\title{
Crecimiento de plantas de maíz (Zea mays) en un suelo contaminado con petróleo y remediado con extracto de cáscaras de naranja (Citrus sinensis)
}

\section{Corn (Zea mays) growth in petroleum contaminated soil, remediated with orange (Citrus sinensis) peel extract}

\author{
Tomás Darío Marín Velásquez
}

\begin{abstract}
Resumen:
La contaminación del suelo genera un fuerte impacto cuando la actividad petrolera se realiza dentro de un ecosistema de sabana. Cualquier derrame afecta suelos que son de vocación agropecuaria. La bioestimulación con extracto de cáscaras de naranja (Citrus sinensis) es una alternativa para la remediación de suelos contaminados con petróleo crudo y en esta investigación se utilizó la planta de maíz (Zea mays) como bioindicador del nivel de contaminación de un suelo de sabana luego de su tratamiento. Se trataron tres muestras de suelo de sabana contaminado con petróleo crudo liviano con disolusiones al 1, 3 y $5 \%$ de extracto de cáscaras de naranja en agua a una dosis de $150 \mathrm{~mL}$ por $\mathrm{kg}$ de suelo. Se midió el contenido de aceites y grasas cada 7 días, hasta un máximo de 42 días. Se plantaron semillas de maíz en las muestras de suelo, se midió su crecimiento cada 5 días por un período de 35 días continuos, comparando su crecimiento con semillas plantadas en una muestra de suelo sin contaminación. Según un análisis de contraste de rangos, el crecimiento de las plantas fue estadísticamente igual en todas las muestras hasta los 20 días, luego se evidenciaron diferencias respecto al patrón.
\end{abstract}

Palabras clave: Citrus sinensis; Zea mays; bioestimulación; contaminación; suelo

\begin{abstract}
:
Soil pollution has a strong impact when oil activity takes place within a savanna ecosystem. Any oil spill affects agricultural soils. Biostimulation with orange peel extract (Citrus sinensis) is an alternative for remediation of soil contaminated with crude oil and in this research the corn plant (Zea mays) was used as a biomarker of contamination level of a savanna soil after their treatment. Three samples of savannah soil contaminated with oil light crude were treated with dissolutions 1,3 and $5 \%$ of extract of orange peel in water at a dose of $150 \mathrm{~mL}$ per $\mathrm{kg}$ of soil treated. The content of oils and fats was measured every 7 days, up to 42 days. Corn seeds were planted in soil samples, their growth was measured every 5 days for a period of 35 consecutive days, comparing their growth with seeds planted in a soil sample without contamination. According to an analysis of rank contrast, the plant growth was statistically the same in all samples up to 20 days; from there, evident differences regarding the pattern were shown.
\end{abstract}

Keywords: Citrus sinensis; Zea mays; bioestimulation; pollution; soil 


\section{Introducción}

La actividad petrolera a nivel mundial está entre las más susceptibles a producir daños al ambiente tanto natural como social, debido a la naturaleza de los hidrocarburos y otros productos químicos que se manejan en las operaciones propias de esta industria. Las consecuencias ambientales de los procesos asociados a la industria petrolera (exploración, perforación, producción, refinación, almacenamiento, transporte, distribución y comercialización) producen graves daños ambientales entre los que se encuentran la deforestación y los cambios del paisaje. "Los principales macroprocesos que tienen lugar en las grandes empresas petroleras son: exploración, perforación, producción, refinación, almacenamiento, transporte, distribución y comercialización. Cada macroproceso genera consecuencias directas sobre el ambiente, inherentes a su naturaleza, entre las que destacan las emisiones atmosféricas, los efluentes líquidos y los desechos sólidos y peligrosos” (Galván et al, 2007, p. 92). El impacto de los derrames petroleros varía de acuerdo con el tipo de crudo transportado, el tamaño del derrame, las condiciones climáticas al momento del derrame y de los ecosistema aledaños (Bravo, 2007, p. 19). La biorremediación está considerada como la alternativa más aplicable para la recuperación de suelos contaminados con petróleo y entre sus variantes la bioaumentación y la bioestimulación han sido ampliamente estudiadas. La bioaumentación, consiste en la introducción al suelo contaminado con hidrocarburos de microorganismos aclimatados o modificados genéticamente con la finalidad que degraden los contaminantes presentes. "Las técnicas de bioaumentación describen la adición de organismos o enzimas a un material con el propósito de eliminar sustancias indeseables" (Shmaefsky, 1999, citado por Ortiz et al, 2005, p.52). La bioestimulación, "se basa en el uso de nutrientes, sustratos o aditivos con actividad superficial para estimular el crecimiento y desarrollo de organismos capaces de biodegradar compuestos contaminantes del medio ambiente" (Ortiz, at al., 2005, p.52).

La investigación se basó en el uso del extracto de la cáscara de naranja dulce (Citrus sinensis) como bioestimulador en un suelo contaminado con petróleo liviano y luego se utilizó la planta de maíz de la variedad reventón (Zea mays everta) como indicador de la calidad del suelo luego del tratamiento, es decir como un bioindicador que se define como "un organismo, o parte de un organismo, o una comunidad de organismos, utilizados para obtener información sobre la calidad del medio ambiente" (Garbisu, et al, 2007, p.45). Se tomaron como variables de estudio el contenido de aceites y grasas según la norma EPA 9071b y el tamaño promedio de las plantas de maíz en las muestras contaminadas y remediadas y en una muestra patrón sin contaminar.

\section{Metodología}

La toma de muestras de suelo se realizó delimitando un área de $35 \mathrm{~m}^{2}$ en una parcela destinada a la siembra de cultivos como maíz (Zea mays) y frijol (Phaseolus vulgaris) ubicada en la población de El Furrial, Estado Monagas, Venezuela. Los suelos de esta población son típicos del 
ecosistema de sabana de los llanos orientales venezolanos y se encuentran enmarcados dentro de un campo productor de petróleo grado liviano - mediano (24 - $\left.32{ }^{\circ} \mathrm{API}\right)$. Para el momento de la toma de muestra, el área no se hallaba cultivada por lo que estaba cubierta de pasto autóctono de la zona y se realizó un muestreo con 10 muestras disgregadas al azar según procedimiento para muestreo de suelos descrito por la norma EPA Method 5035. La muestra de suelo compuesta fue caracterizada para determinar los siguientes parámetros: densidad aparente a través del método del cilindro (Flores y Alcalá, 2010, p.37); densidad real por el método del picnómetro según lo establecido en la norma ASTM D854; textura mediante el método de sedimentación descrito por Cano, (2000); porosidad, calculada a partir de los valores de densidad aparente y real; pH tomando como referencia el procedimiento potenciométrico descrito en la norma ASTM D4972; materia orgánica total por calcinación (Zagal \& Sadzawka, 2007, p. 28) y aceites y grasas por extracción con hexano aplicando el procedimiento establecido en la norma EPA Method 9071b.

La muestra de petróleo crudo utilizada para contaminar el suelo, fue una mezcla de pozos productores del Campo "El Furrial", con una API promedio de 31,1 ํAPI, a la que además se le determinaron las siguientes propiedades: Viscosidad, determinada mediante el uso de un viscosímetro rotacional Brookfield y siguiendo el procedimiento establecido en la norma ASTM D2196; constante Viscosidad - Gravedad, parámetro de caracterización del petróleo que se obtuvo de acuerdo con el procedimiento de cálculo descrito en la norma ASTM D2501; porcentaje de insolubles en n-hexano, se siguió el procedimiento de extracción Soxhlet establecido en la norma ASTM D6560;

La obtención del extracto de cáscaras de naranja (Citrus sinensis) se realizó utilizando como solvente de extracción una mezcla de 70\% alcohol isopropílico y $30 \%$ agua. Las cáscaras de naranja fueron secadas al sol y trituradas en una mezcladora de cuchillas y colocada en una batería de 6 extractores Soxhlet con balones de $500 \mathrm{ml}$ de solvente y $100 \mathrm{~g}$ de cáscaras de naranja por extractor. El extracto fue concentrado en un rotaevaporador con una temperatura de $100 \stackrel{\circ}{\circ}$ y presión atmosférica, para luego preparar la disoluciones al 1, 3 y 5\% del mismo en agua destilada.

Para establecer el grado de contaminación del suelo para el estudio, se diseñó un experimento en el cual se tomaron 5 bandejas de aluminio y en cada una se agregaron $500 \mathrm{~g}$ de suelo y se contaminaron con volúmenes de petróleo de 20,30, 40, 50 y $60 \mathrm{ml}$; se dejaron en reposo durante 7 días y luego a cada una se le plantaron 15 semillas de maíz reventón (Zea mays everta) adicionando una sexta muestra sin contaminar como patrón. Se le agregaron $250 \mathrm{ml}$ de agua a cada muestra. Se midió el tiempo en días necesario para la germinación de las semillas y el porcentaje de germinación, con la finalidad de obtener el volumen mínimo de petróleo letal para el cultivo, es decir aquel en que no se produjera germinación. Este volumen mínimo se tomó como referencia para la etapa de remediación. 
Se tomaron 3 muestras de suelo de $1 \mathrm{~kg}$ cada una y se les contaminó con petróleo de acuerdo con el volumen mínimo determinado en la etapa anterior. Se dejaron en reposo durante 7 días para que el suelo y el petróleo se mezclaran bien. Seguidamente se les aplicó las disoluciones de extracto preparadas a una dosis de $150 \mathrm{~mL} / \mathrm{kg}$ de suelo. Una vez aplicados los productos se tomaron muestras cada 7 días hasta un total de 42 días continuos y se monitorearon los porcentajes de aceites y grasas (\%AyG) en cada período.

Una vez finalizado el proceso de remediación de las muestras contaminadas, se colocaron las mismas en macetas de plástico y en cada una se plantaron 15 semillas de maíz reventón (Zea mays everta). Para tener un patrón de comparación en otra maceta se agregó una muestra de suelo original sin contaminar y en él se plantaron también 15 semillas de maíz. Se regaron las muestras con un volumen de agua constante de $250 \mathrm{ml}$ de forma interdiaria. Se midió la altura de cada planta en cada muestra cada 5 días hasta un total de 35 días, obteniéndose el tamaño promedio, con lo cual se realizó una comparación entre las muestras y de estas respecto al patrón, mediante un ANOVA factorial y prueba de diferencia mínima significativa de Fisher (DMS), para así analizar el efecto del proceso de remediación sobre el desarrollo de las plantas en el suelo.

\section{Resultados}

Resultados obtenidos luego de la caracterización de la muestra compuesta de suelo. En la Tabla 1 se observan los resultados de las propiedades de la muestra de suelo de sabana obtenido de la población de "El Furrial" en el Estado Monagas, Venezuela.

Tabla 1. Propiedades de la muestra de suelo

\begin{tabular}{|l|l|l|l|}
\multicolumn{1}{|c|}{ Propiedad } & Unidad & \multicolumn{1}{c|}{ Valor } & \multicolumn{1}{c|}{ Método } \\
\hline Textura & Adim. & $\begin{array}{l}55 \% \text { Arena } \\
\text { 30\% Arcilla } \\
15 \% \text { Limo }\end{array}$ & Sedimentación \\
\hline Densidad aparente & $\mathrm{g} / \mathrm{mL}$ & 1,2 & Cilindro \\
\hline Densidad Real & $\mathrm{g} / \mathrm{mL}$ & 2,4 & Picnómetro \\
\hline Porosidad & $\%$ & 50 & Calculada \\
\hline pH $(1: 2,5)$ & Adim. & 5,5 & Potenciométrico \\
\hline Materia Orgánica Total & $\%$ & 15,5 & Calcinación \\
\hline Aceite y Grasas & $\%$ & 1,6 & EPA 9071b \\
\hline
\end{tabular}

Resultados obtenidos de la caracterización de la muestra de petróleo crudo del Campo "El Furrial". En la Tabla 2 se observan las propiedades obtenidas de la caracterización de la muestra de petróleo utilizada para contaminar el suelo. 
Tabla 2. Propiedades de la muestra de petróleo

\begin{tabular}{|l|c|c|l|}
\hline \multicolumn{1}{|c|}{ Propiedad } & Valor & Unidad & Método \\
\hline Viscosidad a $26,3^{\circ} \mathrm{C}$ & 10,8 & $\mathrm{cP}$ & ASTM D2196 \\
\hline Viscosidad a $40 \stackrel{\circ}{ }{ }^{\circ} \mathrm{C}$ & 7,6 & $\mathrm{cP}$ & ASTM D2196 \\
\hline VGC & 0,84 & Adm. & ASTM D2501 \\
\hline Insolubles en hexano & 0,2 & $\%$ & ASTM D6560 \\
\hline
\end{tabular}

Resultados del efecto de la contaminación con petróleo sobre la germinación de las semillas de maíz reventón (Zea mays everta). En la Tabla 3 se muestran los resultados obtenidos al realizar un ensayo para determinar la concentración letal del petróleo para las plantas de maíz en el suelo.

Tabla 3. Porcentajes de germinación del maíz reventón (Zea mays everta) en suelos contaminados con petróleo

\begin{tabular}{|c|c|c|c|c|c|c|}
\hline $\begin{array}{c}\text { Petróleo, } \\
\mathrm{mL}\end{array}$ & Suelo, $\mathrm{kg}$ & $\begin{array}{c}\text { Concentración, } \\
\mathrm{mL} / \mathrm{kg}\end{array}$ & $\begin{array}{c}\text { Nro de } \\
\text { Semillas }\end{array}$ & $\begin{array}{c}\text { Plantas } \\
\text { Germinadas }\end{array}$ & Tiempo, días & Germinación, \% \\
\hline 20 & 0,5 & 40 & 15 & 5 & 6 & 33,33 \\
\hline 30 & 0,5 & 60 & 15 & 2 & 6 & 13,33 \\
\hline 40 & 0,5 & 80 & 15 & 1 & 7 & 6,67 \\
\hline 50 & 0,5 & 100 & 15 & 0 & 15 & 0,00 \\
\hline 60 & 0,5 & 120 & 15 & 0 & 15 & 0,00 \\
\hline Patrón & 0,5 & 0 & 15 & 12 & 5 & 80,00 \\
\hline
\end{tabular}

Resultados del proceso de remediación del suelo contaminado con $100 \mathrm{~mL}$ de petróleo por kilogramo. En la Tabla 4, se muestran los resultados del monitoreo del contenido de aceites y grasas en las muestras de suelo contaminadas y tratadas con las disoluciones preparadas con el extracto de cáscaras de naranja dulce (Citrus sinensis) a una dosis de $150 \mathrm{~mL}$ por $\mathrm{kg}$ de suelo contaminado.

Tabla 4. Porcentajes de Aceites y Grasas obtenidos durante el monitoreo de la remediación

\begin{tabular}{|c|c|c|c|}
\multirow{2}{*}{ Tiempo, Días } & \multicolumn{4}{|c|}{ Aceites y Grasas en \% } \\
& $1 \%$ extracto & $3 \%$ extracto & $5 \%$ extracto \\
0 & 9,5 & 9,9 & 9,5 \\
\hline 7 & 6,8 & 6,2 & 4,9 \\
\hline 14 & 4,4 & 3,1 & 3,1 \\
\hline 21 & 3,8 & 2,9 & 2,6 \\
\hline 28 & 2,3 & 2,4 & 1,3 \\
\hline 35 & 2,1 & 1,8 & 1,1 \\
\hline 42 & 1,5 & 1,3 & 0,9 \\
\hline
\end{tabular}

Resultados de la germinación de semillas en las muestras de suelo remediadas y en la muestra patrón. En la Tabla 5 se muestran los resultados del porcentaje de germinación de las semillas de maíz reventón (Zae mays everta) plantadas en las muestras de suelo luego de la remediación en comparación con las germinadas en la muestra patrón. 
Tabla 5. Porcentaje de germinación de las semillas en las muestras de suelo remediadas

\begin{tabular}{|c|c|c|c|}
\hline Muestra & Semillas plantadas & Semillas germinadas & Germinación, \% \\
\hline Patrón & 15 & 15 & 100,0 \\
\hline Remediada con 1\% de extracto & 15 & 14 & 93,3 \\
\hline Remediada con 3\% de extracto & 15 & 13 & 86,7 \\
\hline Remediada con 5\% de extracto & 15 & 13 & 86,7 \\
\hline
\end{tabular}

Resultados del crecimiento promedio en centímetros de las plantas de maíz reventón (Zea mays everta). En la Tabla 6 se observan los resultados del tamaño promedio de las plantas de maíz en las muestras de suelo remediadas con las disoluciones de extracto de cáscaras de naranja a una dosis de $150 \mathrm{~mL}$ por $\mathrm{kg}$ de suelo y en una muestra de suelo original sin contaminar utilizado como patrón de comparación.

Tabla 6. Crecimiento promedio de las plantas de maíz

\begin{tabular}{|c|c|c|c|c|}
\hline \multirow{2}{*}{ Tiempo, Días } & \multicolumn{4}{|c|}{ Altura en cm } \\
\cline { 2 - 5 } & $1 \%$ de extracto & 3\% de extracto & $5 \%$ de extracto & Patrón \\
\hline 5 & 10,0 & 6,9 & 7,1 & 11,7 \\
\hline 10 & 15,2 & 13,7 & 12,9 & 20,7 \\
\hline 15 & 19,7 & 20,7 & 16,7 & 25,2 \\
20 & 23,1 & 22,0 & 17,8 & 32,0 \\
25 & 24,4 & 22,6 & 24,8 & 47,7 \\
30 & 26,4 & 30,3 & 27,0 & 52,8 \\
35 & 27,0 & 31,0 & 30,0 & 55,8 \\
\hline
\end{tabular}

Resultados del análisis de varianza de dos factores (ANOVA Factorial) aplicado a los resultados obtenidos del crecimiento promedio de las plantas. En la Tabla 7 se muestran los resultados obtenidos a partir del ANOVA Factorial en el cual se toman cono factores o variables independientes el tiempo y las muestras y la variable dependiente el crecimiento promedio de las plantas. El análisis se realizó mediante el programa LibreOffice 4.4 Calc.

Tabla 7. Resultados del ANOVA Factorial

\begin{tabular}{|c|c|c|c|c|c|c|}
\hline Fuente de Variación & Suma de cuadrados & GL & Cuadrado medio & F & P-Valor & F crítico \\
\hline Tiempo & 2355,78 & 6 & 392,63 & 14,44 & 0,00 & 2,66 \\
Muestra & 1142,32 & 3 & 380,77 & 14,00 & 0,00 & 3,16 \\
\hline Error & 489,42 & 18 & 27,19 & \multicolumn{2}{|l}{} \\
Total & 3987,51 & 27 & \multicolumn{3}{|l|}{}
\end{tabular}

Resultados de la prueba de diferencia mínima significativa de Fisher. En la Tabla 8 se observan los resultados de la prueba de contraste múltiple de rangos basada en la diferencia mínima significativa de Fisher realizada mediante el programa LibreOffice 4.4 Calc. Los valores identificados con letras iguales son estadísticamente iguales. 
Tabla 8. Contraste de Rangos por DMS de Fisher para el tamaño promedio de las plantas

\begin{tabular}{|c|c|c|c|c|}
\hline Tiempo, Días & $1 \%$ de extracto & $3 \%$ de extracto & $5 \%$ de extracto & Patrón \\
\hline 5 & A & A & A & A \\
\hline 10 & A & A & A & A \\
\hline 15 & A & A & A & A \\
\hline 20 & A & A & A & A \\
\hline 25 & A & A & A & B \\
\hline 30 & A & A & A & B \\
\hline 35 & A & A & A & B \\
\hline DMS (Muestra) $=12,0 \mathrm{~cm}$ & & & \\
\hline DMS (Tiempo) $=12,9 \mathrm{~cm}$ &
\end{tabular}

En la Figura 1 se observa el comportamiento gráfico del crecimiento promedio de las plantas de maíz reventón (Zea mays everta) durante el período de monitoreo de 35 días continuos en las muestras de suelo remediadas con las disoluciones de extracto de cáscaras de naranja (Citrus sinensis) y en la muestra patrón original sin contaminación.

\section{Discusión}

En la Tabla 1 se pueden observar las principales características del suelo utilizado para el estudio. El suelo posee buenas características para uso agrícola, ya que posee una cantidad muy alta de materia orgánica, esto según lo que establece Rioja (2002) citado por Pavón (sf) el cual indica que cantidades de MOT mayores a 3,6\% en suelos de clima cálido se clasifica como muy alta. Este suelo se puede clasificar como de tipo Molisol, que es un suelo propio de pradera donde la descomposición de las hojas de las gramíneas induce la gran concentración de materia orgánica como lo reseña la Soil Survey Staff (2010). Posee una textura franco arenosa arcillosa que le da propiedades de alta porosidad (50\%) con densidades propias de suelo de textura fina (Flores y Alcalá, 2010). El pH indica que se trata de un suelo de características ácidas con pH de 5,5 fuertemente ácido, lo cual es característico de las sabanas venezolanas, sobre todo en los llanos orientales donde se observan bajos valores de esta propiedad (López-Hernández \& Ojeda, 1996). 


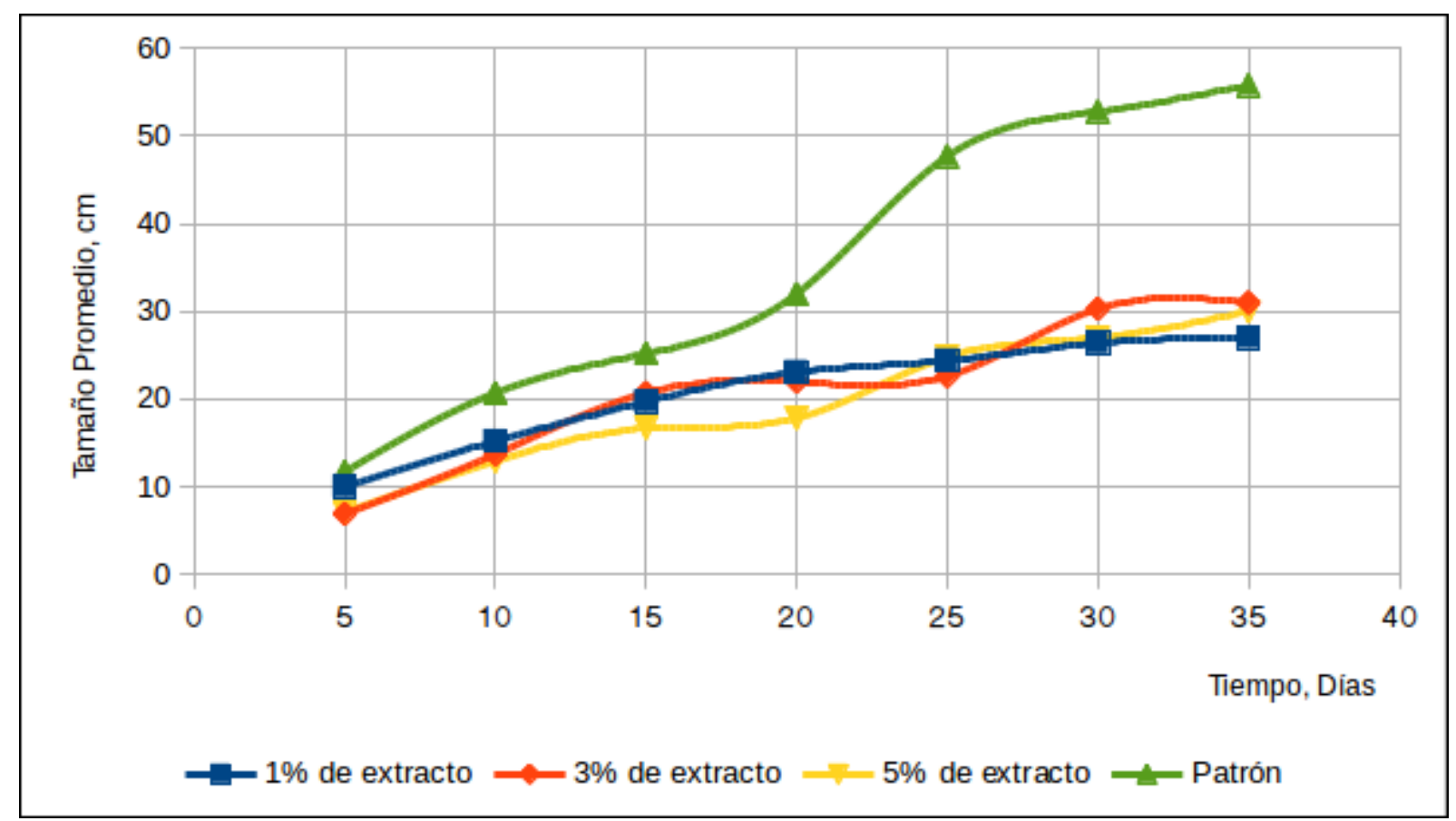

Figura 1. Gráfico del crecimiento promedio de las plantas de maíz

El contenido de aceites y grasas aunque está por encima del valor máximo permitido para el suelo que es de $1 \%$ según norma venezolana Decreto 2635, puede estar influenciado por el contenido de materia orgánica, ya que esta propiedad se refiere a cualquier aceite de origen animal, vegetal o mineral que esté presente en el suelo y que puede ser extraído con hexano, según lo establecido en la norma EPA Method 9071b.

En la Tabla 2 se muestran las características principales de la muestra de Petróleo Crudo. Según su Gravedad API, se clasifica como Petróleo Liviano o Ligero (31,1 ํAPI) con una buena viscosidad para su transporte y procesamiento $(10,5 \mathrm{cP}$ a $26,3 \stackrel{\circ}{\circ})$ con una composición estimada de crudo mixto con tendencia a Nafténico según lo que establece la Norma Técnica Colombiana NTC2731, al tomar como referencia el valor de VGC de 0,84. El contenido de insolubles en nhexano indica la cantidad de componentes pesados (tipo asfaltenos) presente en la composición del crudo, lo cual es una característica de suma importancia para el estudio de biodegradabilidad, debido a que estos compuestos pesados son difíciles de degradar por los microorganismos del suelo, además se debe tomar en cuenta este porcentaje $(0,2 \%)$ debido a que este es el límite de $\% A y G$ que se puede obtener al usar el n-hexano como solvente.

Según los resultados mostrados en la Tabla 3, la germinación de las semillas de maíz se vio afectada de forma directa por la cantidad de petróleo utilizado para contaminar el suelo. A mayor cantidad de petróleo menor fue el porcentaje de germinación, obteniéndose que a partir de $50 \mathrm{~mL}$ no se produjo germinación alguna. Este resultado indica que a partir de una contaminación con 50 $\mathrm{mL}$ de crudo por cada $0,5 \mathrm{~kg}$ de suelo $(8,6 \% \mathrm{p} / \mathrm{p})$ las propiedades del suelo se alteran (sobre todo el $\mathrm{pH}$ ) de tal manera que no permiten la germinación y desarrollo del maíz. El efecto de la contaminación por petróleo en el tiempo de germinación, no es significativa hasta la dosis de 30 
$\mathrm{mL}$ por cada $0,5 \mathrm{~kg}$ de suelo $(5,2 \% \mathrm{p} / \mathrm{p})$ en comparación con el patrón, esto según un análisis ANOVA con el cual se demostró que la mínima diferencia significativa fue de 1,88 días; el efecto se observó solo para la dosis de $40 \mathrm{~mL}$ por cada $0,5 \mathrm{~kg}$ de suelo $(7,0 \% \mathrm{p} / \mathrm{p})$ cuyo valor es estadísticamente igual al tiempo de germinación para las otras dosis de petróleo, pero diferente al patrón. Este resultado coincide con el de Méndez-Natera et al (2004) en una investigación donde se reportó que no hubo diferencias significativas en el tiempo de germinación de semillas de maíz (Zea mays) cv. Himeca 95 en muestras de suelo de sabana contaminado con petróleo sembradas una semana después de la contaminación con concentraciones de 3 y $6 \%$ p/p, aunque en esta investigación no se reportan las características del petróleo utilizado.

En la Tabla 4 se muestran los resultados del monitoreo de la remediación con $150 \mathrm{~mL}$ de extracto de cáscaras de naranja dulce por kilogramo de suelo contaminado durante un periodo de 42 días continuos, llegándose a porcentajes de disminución de 84,2\% para la disolución al 1\%; 86,9\% para la disolución al 3\% y 90,5\% para la disolución al 5\%. Según lo establece el decreto 2635 de las normas ambientales venezolanas un suelo se considera descontaminado cuando su contenido de Aceites y Grasas en menor o igual al 1\% y considerando que el suelo originalmente contenía $1,6 \%$ de AyG entonces el tratamiento fue efectivo para todas las disoluciones a partir del día 28 , llegándose incluso a obtenerse valores menores de este parámetro en comparación con el suelo original. La efectividad del tratamiento aumenta con la cantidad de extracto en la disolución y al aplicar un análisis de diferencia mínima significativa se demostró que existe diferencia entre los porcentajes de disminución de los Aceites y Grasas, siendo la DMS de 1,99\%.

Al igual que lo observado en la Tabla 3, en la Tabla 5 se muestran los resultados del porcentaje de germinación de las semillas de maíz sembradas luego del proceso de tratamiento, obteniéndose que no existen diferencias significativas entre el número se semillas germinadas en las muestras de suelo remediadas. En la muestra patrón se obtuvo 100\% de germinación, lo cual no tiene diferencia estadística con el porcentaje obtenido para las semillas sembradas en la muestra remediada con la disolución al 5\%, pero mostró diferencias respecto a los resultados obtenidos en las muestras remediadas con 1 y $3 \%$ de disolución.

En la Tabla 6 se observan los resultados del crecimiento promedio de las plantas de maíz en las muestras remediadas respecto a la muestra patrón. Se observa que las plantas en la muestra patrón para cada día de monitoreo son de mayor tamaño que las plantas en las muestras de suelo remediadas. El ANOVA factorial (Tabla 7 ) muestra que existe influencia estadísticamente significativa de los dos factores en estudio (tiempo y muestra) sobre la altura promedio de las plantas, lo que significa que tanto el tiempo como la muestra de suelo contaminado y remediado son factores importantes para el desarrollo de las plantas de maíz. El análisis por diferencia mínima significativa muestra según lo que se observa en la Tabla 8 que el crecimiento promedio en los días de monitoreo para las plantas sembradas en las muestras de suelo remediadas es 
igual desde el punto de vista estadístico durante todo el tiempo de ensayo; este resultado está en concordancia con lo obtenido por Quiñónez-Aguilar et al (2008) donde se muestra que las plantas de maíz crecen de forma estadísticamente igual en suelos con concentraciones de petróleo liviano de $15000 \mathrm{mg} / \mathrm{kg}, 25000 \mathrm{mg} / \mathrm{kg}$ y $35000 \mathrm{mg} / \mathrm{kg}$ lo que corresponde a $1,5 \% ; 2,5 \%$ y $3,5 \% \mathrm{p} / \mathrm{p}$ de petróleo en el suelo.

En la Tabla 8 también se observa que al comparar el crecimiento promedio de las plantas de maíz en los suelos remediados con las plantas en el suelo patrón, el mismo es igual hasta el día 20, a partir del cual se evidencia diferencia significativa favorable al crecimiento de las plantas en el suelo patrón. Un resultado similar fue reportado por Quiñónez-Aguilar et al (2008) en donde las plantas cultivadas en el suelo sin contaminar superaron significativamente a las plantas de los suelos contaminados $(15000,25000$ y $35000 \mathrm{mg} / \mathrm{kg}$ de suelo) respecto al valor de la altura de la parte aérea y que independientemente de la cantidad de petróleo en el suelo, el crecimiento de las plantas fue estadísticamente igual en presencia de la contaminación.

El comportamiento gráfico del crecimiento de las plantas de maíz se observa en la Figura 1, donde se corrobora que hasta el día 20 todas las plantas siguen una tendencia regular y similar, sobre todo en las muestras de suelo remediadas. A partir de este tiempo se observa como las plantas en la muestra patrón cambian su desarrollo y se alejan de la tendencia original, lo cual coincide con el cambio en la significancia estadística mostrada en la Tabla 8. Beltrán (2014) obtuvo para dos tipos de maíz, uno de variedad y uno híbrido un crecimiento basado en la altura de $21 \mathrm{~cm} \mathrm{y} 18 \mathrm{~cm}$ respectivamente para el mismo período (35 días) en suelos que fueron fertilizados y bajo condiciones óptimas, resultado inferior a los obtenidos en este estudio incluso para los suelos remediados, lo cual demuestra la eficiencia de los tratamientos y la calidad del suelo en estudio. Este resultado se puede deber a que la cantidad alta de materia orgánica presente en el suelo original deja luego de la remediación una cantidad suficiente para el desarrollo de las plantas, lo que también influyó en el mayor desarrollo de las plantas en el suelo patrón, las cuales superaron lo obtenido por Beltrán (2014) en cuyo estudio las plantas necesitaron entre 56 y 63 días para alcanzar alturas equivalentes a las máximas obtenidas en el suelo patrón a los 35 días.

\section{Conclusiones y Recomendaciones}

De acuerdo con los resultados obtenidos se puede concluir lo siguiente:

La concentración letal del Petróleo utilizado en el suelo para la germinación de las plantas de maíz reventón (Zea mays everta) fue de $8,6 \% \mathrm{p} / \mathrm{p}$.

Las muestras de suelo contaminadas con el Petróleo fueron remediadas de manera efectiva al aplicar las disoluciones $1 \%, 3 \%$ y $5 \%$ de extracto de cáscaras de naranja dulce (Citrus sinensis) en agua. 
Una vez remediadas las muestras de suelo contaminadas con el Petróleo, las semillas de maíz reventón (Zea mays everta) lograron germinar sin diferencias significativas entre el número de semillas germinadas en cada una de las muestras de suelo.

Los resultados del crecimiento promedio de las plantas de maíz reventón en las muestras de suelo remediadas fue estadísticamente igual durante todo el período de monitoreo de 35 días, el desarrollo de las plantas es igual en todas las muestras incluida la de suelo sin contaminar, iguales hasta los 20 días, a partir de los cuales se evidenció un crecimiento en la muestra patrón superior y estadísticamente diferente a los demás.

Se corroboró la efectividad de la remediación aplicando extracto de cáscaras de naranja dulce (Citrus sinensis) basándose en el crecimiento de las plantas de maíz; las diferencias observadas producto de los cambios en el suelo producto del proceso de biorremediación.

\section{Bibliografía}

ASTM D854. (2014). Standard Test Methods for Specific Gravity of Soil Solids by Water Pycnometer. West Conshohocken, USA. ASTM International.

ASTM D2196. (2015). Standard Test Methods for Rheological Properties of Non-Newtonian Materials by Rotational Viscometer. West Conshohocken, USA. ASTM International.

ASTM D2501. (2014). Standard Test Method for Calculation of Viscosity-Gravity Constant (VGC) of Petroleum Oils. West Conshohocken, USA. ASTM International.

ASTM D4972. (2013). Standard Test Method for pH of Soils. West Conshohocken, USA. ASTM International.

ASTM D6560. (2012). Standard Test Method for Determination of Asphaltenes (Heptane Insolubles) in Crude Petroleum and Petroleum Products. West Conshohocken, USA. ASTM International.

Beltrán, J. (2014). Informe de crecimiento y desarrollo (cultivo de maíz) (fisiología vegetal). Recuperado de http://mcjabe.blogspot.com/

Bravo, E. (2007). Los impactos de la explotacion petrolera en ecosistemas tropicales y la biodiversidad. Acción Ecológica. Recuperado de http://www.inredh.org/archivos/.../impactos explotacion petrolera esp.pdf

Flores, L. \& Alcalá, J. (2010). Manual de Procedimientos Analíticos. Laboratorio de Física de Suelos. Universidad Nacional Autónoma de México. Instituto de Geología. Departamento de Edafología.

Recuperado de 
http://www.geologia.unam.mx/igl/deptos/edafo/lfs/MANUAL\%20DEL\%20LABORATORIO\%2 ODE\%20FISICA\%20DE\%20SUELOS1.pdf

Galván, L., Guédez, C., Reyes, R. \& De Armas, D. (2007). Los macroprocesos de la industria petrolera y sus consecuencias ambientales. Revista Universidad, Ciencia y Tecnología. 11(43), 91-97

Garbisu, C., Becerril, J., Epelde, L. \& Alkorta, I. (2007). Bioindicadores de la calidad del suelo: herramienta metodológica para la evaluación de la eficacia de un proceso fitorremediador. Revista Ecosistemas. 16(2), 44-49

López-Hernández, D. \& Ojeda, A. (1996). Alternativas en el manejo agroecológico de los suelos de las sabanas del norte de Sudamérica. Revista Ecotrṕicos. 9(2), 101-117

Méndez-Natera, J., Roque, C., Zapata, K. \& Otahola-Gómez, V. (2004). Efecto de la concentración y tiempo de contaminación de un suelo por petróleo en la germinación de semillas de maíz (Zea mays L.) cv. Himeca 95. Revista UDO Agrícola. 4(1), 66-71

METHOD 5035. (2004). Methodologies for collection, preservation, storage, and preparation of soils to be analyzed for volatile organic compounds. United States Environmental Protection Agency (EPA). State of California. USA

METHOD 9071B. (1998). N-hexane extractable material (hem) for sludge, sediment, and solid samples. United States Environmental Protection Agency (EPA). State of California. USA

NORMA TÉCNICA COLOMBIANA NTC 2731. (2001). Caucho. Aceites de petróleo plastificantes para uso en la industria del caucho. Instituto Colombiano de Normas Técnicas y Certificación (ICONTEC). Bogotá, Colombia.

Ortiz, E., Núñez, R., Fonseca, E., Oramas, J., Almazán, V., Cabranes, Y., Miranda A., Barbán O., Martínez, C., Díaz, Y. \& Borges, G. (2005). Biorremediacion de suelos contaminados con hidrocarburos. Revista Contribución a la Educación y la Protección del Medio Ambiente. 6, $51-60$

Pavón, A. (sf). Instalación de riego por goteo en una parcela de maíz. Anejo III: Análisis de suelo. Universidad Ciudad Real. Ciudad Real, España.

Quiñonez, E., Ferrera, R., Gavi, F., Fernández, L., Rodríguez, R. \& Alarcón, A. (2003). Emergencia y crecimiento de maíz en un suelo contaminado con petróleo crudo. Revista Agrociencia. 37(6), 585-594 
Soil Survey Staff. (2010). Claves para la Taxonomía de Suelos. Departamento de Agricultura de los Estados Unidos Servicio de Conservación de Recursos Naturales (USDA). Washington, DC, USA.

Zagal, E. \& Sadzawka, A. (2007). Protocolo de métodos de análisis para suelos y lodos. Universidad de Concepción Facultad de de Agronomía. Chillán, Chile. 